\title{
Analysis of the bioavailability of tea species
}

\author{
Pavel Maslennikov*, Mikhail Zorin, and Anna Seregina \\ Immanuel Kant Baltic Federal University, Str. Universitetskaya, 2, 236040 Kaliningrad, Russian \\ Federation
}

\begin{abstract}
In samples of different tea brands the content of biologically active substances (total water-soluble antioxidants content, ascorbic acid, rutin, polyphenols, anthocyanins) was determined. The water-soluble antioxidant biological availability of the studied tea samples was evaluated. Green Tea with Lemon and Ceylon Baikhovi Black Spiced Tea is characterized by similar levels of antioxidant bioavailability. After the consumption of both beverages, there was a definite increase in the content of antioxidants in saliva of individuals with an initially low content of antioxidants. Amaranth Phytotea antioxidants were characterized by low level of bioavailability. The consumption of this beverage did not change the level of antioxidants in saliva, regardless of their initial content.
\end{abstract}

\section{Introduction}

Over the recent decades, the experts in the field of biology and medicine have paid increasing attention to the problem of oxidative stress and ways to prevent it. Reactive oxygen intermediates have been shown to have a toxic effect on the body by damaging membrane lipids, proteins and DNA. Animal and plant organisms have special protective mechanisms antioxidant systems, the components of which are capable to bind the resulting excess radicals, preventing the development of oxidative stress [1-3].

The formation of reactive oxygen intermediates can occur both in normal aerobic metabolism and in exogenous physical and chemical factors, directly or indirectly generating reactive oxygen intermediates. The increasing negative impact of the environment on modern humans increases the relevance of developing new ways to prevent oxidative stress [1-3].

Over the past few years, there is a strong focus on the antioxidant activity of a number of food stuff, primarily of plant products, due to the presence of polyphenols, including flavonoids and tannins [4]. These compounds have the ability to directly inhibit free radicals and chelate metals, including ferrum, thereby producing a marked antioxidant effect [5].

Tea is a popular food consumed by the vast majority of the population and is rich in plant antioxidants [6]. Currently, a variety of tea types as well as herbal tea spices are available to consumers. Although the healing properties of tea have been known since antiquity, the study of the biochemical and pharmacological properties of tea collections using the methodological capabilities of modern science is of great theoretical and practical importance.

\footnotetext{
* Corresponding author: pashamaslennikov@mail.ru
} 
In this context, the purpose of this work is to study the bioavailability of plant antioxidants contained in tea species. Analysis of biologically active substances (ascorbic acid, rutin, polyphenols, anthocyanins, water-soluble antioxidants) in different types of tea.

\section{Materials and methods}

The content of biologically active compounds in samples of seven types of tea was determined: Flower Tea (Sudanese rose, Egypt), Green Tea with Lemon (Lemon Gree Tee, Winckler GmbH, Germany), Amaranth Phytotea (Russia), Ceylon Baikhovi Black Spiced Tea (Greenfield tea Ltd, England), Marco Tea Loose Leaf Green Tea (Venture Tea Ltd, Sri Lanka), Baikhovi Small Leaf Black Tea with Bergamot Aroma (Ahmad Tea Ltd, England), Mint Herbal Tea (Kaliningrad Region, Russia).

The content of anthocyanin pigments was determined in $1 \%$ aqueous extract of hydrogen chloride; the homogenate was previously centrifuged for $30-45$ minutes at $4,500 \mathrm{rpm}$. The anthocyanin total content was calculated using the formula using the specific absorption index of cyanidin-3,5-diglycoside in 1\% aqueous hydrochloric acid solution (453). The absorption of these pigments was determined on a UV-3600 (Shimadzu, Japan) spectrophotometer at a wavelength of $510 \mathrm{~nm}[7,8]$.

The carotenoids were quantified using a spectrophotometric method without prior separation in $100 \%$ acetone extract, followed by calculation according to a Holm equation. To calculate the concentration, the extract optical density was determined at wavelengths of 440, 644, $662 \mathrm{~nm}[8]$.

Ascorbic acid was quantified using the titration method [5]. The principle of the vitamin $\mathrm{C}$ quantitation method is based on its ability to reduce 2,6-dichlorophenolindophenol, which is blue in an alkaline medium and colorless in a reduced state.

The quantitative level of rutin was determined by the Cushman titrate method [5]. The determination of rutin is based on its ability to oxidize with permanganate. Indigo carmine was used as an indicator, which reacts with permanganate after all rutin is oxidized.

The total water-soluble antioxidants content (TWAC) was determined by the amperometric method on a Color Yauza 01-AAA device according to the Yashin Ya.I. method [9].

The study investigated the antioxidant bioavailability of three tea species - Green Tea with Lemon (Lemon Gree Tee), Ceylon Baikhovi Black Spiced Tea (Greenfield) and Amaranth Phytotea. To determine the antioxidant bioavailability, saliva was collected from 30 volunteers. The volunteers were a homogeneous group of women aged from 18 to 21 . Saliva was sampled in the fasted state (3-4 hours after meals). The mouth had to be rinsed with distilled water 2-3 times before sampling. The collected samples were centrifuged or vortexed. The supernatant liquid was quantitatively transferred to the volumetric tube and diluted with the eluent in a ratio of 1:50 (100 mcl of saliva sample up to $5 \mathrm{ml}$ of eluent). The total water-soluble antioxidants content was further analyzed. To assess the antioxidant bioavailability in tea samples, saliva was taken before drinking tea and after 60 minutes.

Statistical processing of the received data was carried out in Statistica ver. program 12 (Statsoft Inc., Thulsa, OK, USA). To identify statistically valid differences between the experimental options, the data were processed using single-factor analysis of variance (ANOVA). The Tukey's HSD test was used as the criterion for the validity of the differences at a significance value $p<0.05$. A preliminary Shapiro-Wilk test was performed to test the normal distribution of data, which did not reveal any obstacles for applying analysis of variance. The correlation degree between the two variables was estimated using the Pearson correlation coefficient (r-Pearson). The tables contain statistically processed data in the form of arithmetic averages and their standard errors. 


\section{Results and Discussion}

The work presents the results of a comparative analysis of the content of the most important biologically active compounds with antioxidant activity (total water-soluble antioxidants content, ascorbic acid, rutin, carotenoids, anthocyanines) as well as their bioavailability in tea compositions presented in the retail trade system. It should be noted that among the selected tea samples, green tea, black tea and herbal collections of various compositions were present. It is shown that tea species differed significantly in the content of biologically active substances, which is related to the peculiarities of their composition. The antioxidant content in the tea leaf is known to be determined by a number of factors, including agricultural and technological [10]. The maximum water-soluble antioxidants content was found in green tea samples (Table 1), which is well consistent with the numerous indications that green tea has the most pronounced antioxidant properties, not fermented during processing [11]. Phenolic compounds are the most common group of secondary plant metabolites and perform a wide range of physiological functions, including active participation in oxidative and restorative reactions. It is important that water-soluble phenol compounds are largely preserved after heat treatment [11], thus tea containing high concentrations of phenolic compounds retain their antioxidant properties during infusion.

All analyzed green and black tea species contained a similar amount of rutin, the glycoside of the flavonoid quercetin, which has P-vitamin activity, but herbal species varied considerably on this parameter. Minimal content of rutin was observed in the Amaranth Phytotea and in Sudanese rose Flower Tea. On the other hand, the Mint Herbal Tea was characterized by a very high content of rutin. The large differences in the content of rutin in the different herbal species are probably related to the characteristics of their composition [12]. In particular, there is an evidence that tea composition additives can significantly change the rutin level [12]. Thus, adding lemon almost doubles the vitamin P content. Since mint contains a significant amount of rutin (according to some data, up to $13.8 \mathrm{mg} \%$ ), it is not surprising that tea species based on it leads in its content.

Herbal tea is the leading source of ascorbic acid, which is 2-2.5 times higher than that of green and black teas. The content of ascorbic acid obtained by us for green and black teas is consistent with that of other authors $[13,14]$. Since the absorption of vitamin P and ascorbic acid is better in a complex, we should especially mention Mint Herbal Tea, which among the samples studied by us contained the maximum quantity of both rutin and ascorbic acid.

The carotenoid content of the studied tea compositions also varied significantly. Interestingly, we found a significant difference in this indicator between green teas. Green Tea with Lemon contained 3.6 times less carotenoids than Marco Tea Green Tea. This may be due to the fact that carotenoids, which are unstable compounds, are easily oxidized by atmospheric air, and are better preserved in whole leaf teas.

Table 1. Antioxidant content in various tea and herbal species.

\begin{tabular}{|l|c|c|c|c|c|}
\hline $\begin{array}{l}\text { Tea species } \\
\text { type }\end{array}$ & $\begin{array}{c}\text { Total Water- } \\
\text { Soluble } \\
\text { Antioxidants } \\
\text { Content, mg } \\
\mathbf{Q E ~ g}^{-1}\end{array}$ & $\begin{array}{c}\text { Rutin, } \\
\mathbf{m g} \%\end{array}$ & $\begin{array}{c}\text { Ascorbic } \\
\mathbf{\text { acid, }} \\
\mathbf{m g} \%\end{array}$ & $\begin{array}{c}\text { Carotenoids, } \\
\boldsymbol{\mu g ~ g}^{-1}\end{array}$ & $\begin{array}{c}\text { Anthocyanins, } \\
\text { \% }\end{array}$ \\
\hline Flower tea & $21.67 \pm 1.24$ & $8.12 \pm 0.72$ & $42.2 \pm 4.2$ & $59.4 \pm 5.6$ & $0.45 \pm 0.04$ \\
\hline $\begin{array}{l}\text { Green Tea with } \\
\text { Lemon }\end{array}$ & $39.01 \pm 2.54$ & $23.41 \pm 1.92$ & $40.1 \pm 3.5$ & $68.41 \pm 7.1$ & $0.41 \pm 0.04$ \\
& & & & & \\
\hline
\end{tabular}


Table 1. Continued.

\begin{tabular}{|l|c|c|c|c|c|}
\hline $\begin{array}{l}\text { Amaranth } \\
\text { Phytotea }\end{array}$ & $8.25 \pm 0.87$ & $7.16 \pm 0.92$ & $20.1 \pm 1.8$ & $110.5 \pm 10.2$ & $0.23 \pm 0.02$ \\
\hline $\begin{array}{l}\text { Ceylon } \\
\text { Baikhovi Black } \\
\text { Spiced Tea }\end{array}$ & $22.69 \pm 1.89$ & $23.40 \pm 2.14$ & $18.4 \pm 1.6$ & $287.4 \pm 25.2$ & $0.37 \pm 0.03$ \\
\hline $\begin{array}{l}\text { Marco Tea } \\
\text { Loose Leaf }\end{array}$ & $36.13 \pm 3.12$ & $24.65 \pm 2.14$ & $19.7 \pm 1.9$ & $246.3 \pm 23.1$ & $0.31 \pm 0.03$ \\
$\begin{array}{l}\text { Green Tea } \\
\text { Laikhovi Small Black Tea } \\
\text { with Bergamot }\end{array}$ & $30.11 \pm 2.58$ & $22.12 \pm 2.03$ & $22.4 \pm 2.1$ & $275.4 \pm 24.5$ & $0.19 \pm 0.02$ \\
Aroma & $10.67 \pm 1.05$ & $40.2 \pm 4.12$ & $48.9 \pm 4.2$ & $82.7 \pm 8.2$ & $0.15 \pm 0.01$ \\
\hline $\begin{array}{l}\text { Mint Herbal } \\
\text { Tea }\end{array}$ & & & & & \\
\hline
\end{tabular}

Data are expressed as mean values \pm standard deviations. Differences were considered significant at $\mathrm{p} \leq 0.05$.

The level of anthocyanins in studied teas did not depend on the tea type. The maximum quantity of anthocyanins was found in tea containing Sudanese rose flowers (Hibiscus), which contain high levels of anthocyanins. Significant differences in anthocyanin content between the analyzed black tea and green tea may be related to the processing characteristics of the tea leaf, in particular during the drying process. There is evidence that anthocyanin content strongly reduces when the samples are dried. So, when blueberries are dried in the air without direct access to sunlight, their losses are about $40 \%$, and in some samples of dried blueberries purchased at the pharmacy, they reached $60 \%$. No correlation between carotenoids and anthocyans was found in the analyzed teas, although there is an evidence in the literature of a positive correlation between the mean conjugation between anthocyanin content and carotenoid level in the plant leaves of the coastal dune complex [15]. It can therefore be assumed that the correlation between anthocyanins and carotenoids may be determined by plant climatic conditions. A cluster analysis of tea species on the content of special antioxidants was carried out. The analysis results allowed to divide all analyzed tea samples into two groups, depending on their phytochemical value (Fig. 1). 


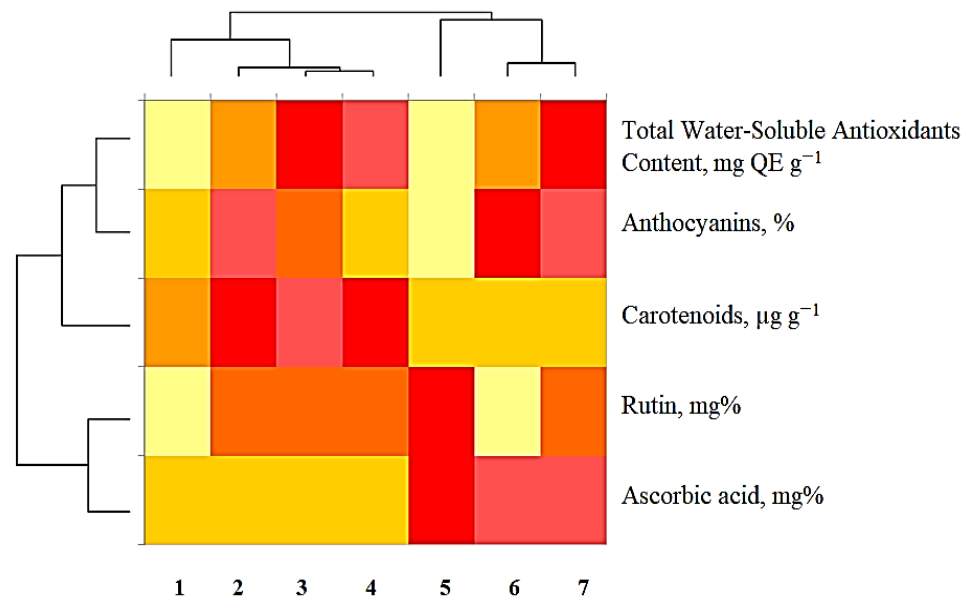

Fig. 1. Heat map (dark - high value, light - low value) with clusters for classifying extracts of different types of tea species according to their content of different antioxidants.

(1) Amaranth Phytotea; (2) Ceylon Baikhovi Black Spiced Tea; (3) Loose Leaf Green Tea; (4) Baikhovi Small Leaf Black Tea with Bergamot Aroma; (5) Mint Herbal Tea; (6) Flower Tea; (7) Green Tea with Lemon.

The bioavailability research was performed for samples of three tea species - Green Tea with Lemon, Ceylon Baikhovi Black Spiced Tea and Amaranth Phytotea (Russia). In the course of the experiment, the subjects were conditionally divided into two groups: persons with an initially high level of antioxidant saliva activity $(0.22 \pm 0.02 \mathrm{mg} / \mathrm{ml})$ and persons with low level of antioxidant saliva activity $(0.035 \pm 0.005)$. As a criterion of bioavailability, the change in the level of the total content of antioxidants in the saliva of volunteers was estimated 60 minutes after drinking. It was shown that after drinking green and black tea, the level of antioxidants in the volunteers' saliva with the initial low antioxidant activity was credibly increased (more than twice). After drinking Amaranth Phytotea no similar effect was observed. Thus, we can conclude about a higher degree of antioxidant bioavailability in the green and black tea composition. An interesting fact is that the total amount of antioxidants in saliva has decreased after the drinking green and black tea in persons with initially high antioxidant activity. Perhaps this pattern is associated with complex mechanisms of absorption and assimilation of antioxidant compounds in the human body. 


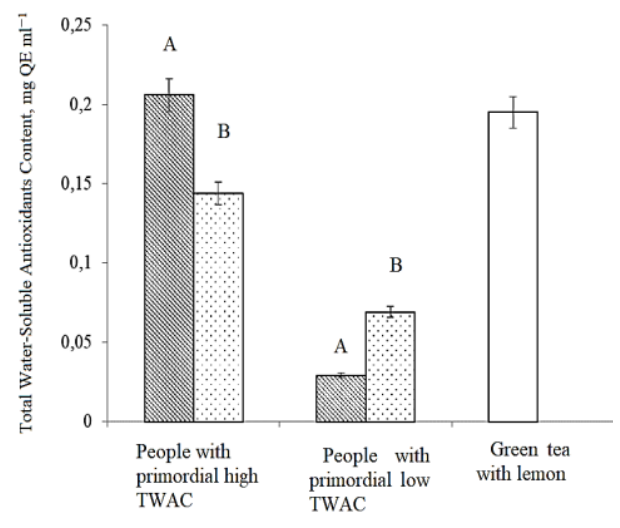

(1)

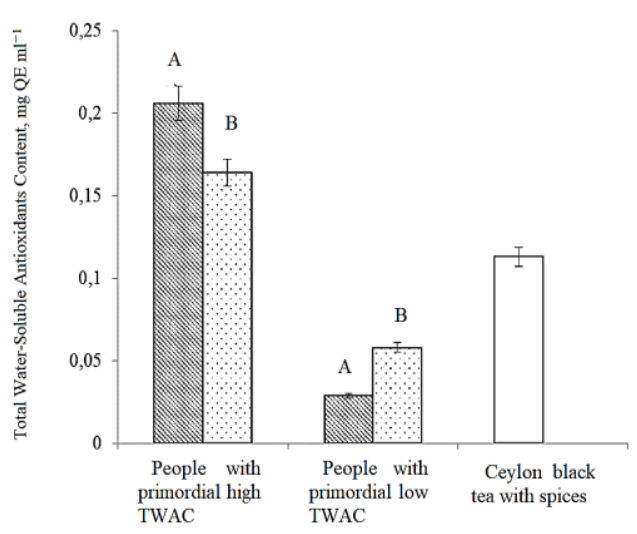

(2)

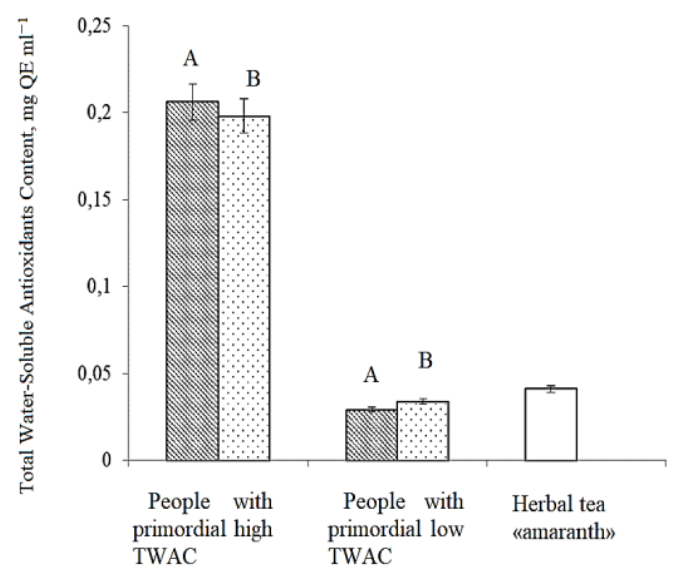

(3)

Fig. 2. Antioxidant bioavailability of Green Tea with Lemon (1), Ceylon Baikhovi Black Spiced Tea (2), Amaranth Phytotea (3). A- TWAC before drinking beverage; B - TWAC after.

\section{Conclusion}

The studied samples of tea and herbal species differ significantly in the content of phenolic antioxidants. The maximum quantity of antioxidants is characteristic for samples of green tea, the minimum - for the Amaranth Phytotea and Mint Herbal Tea. All studied green and black tea species contain a similar amount of rutin, while herbal species vary significantly. The minimum amount of rutin is characteristic for Amaranth Phytotea and Sudanese rose Flower Tea, the maximum is for Mint Herbal Tea. The maximum amount of ascorbic acid among the studied tea compositions is contained in the Mint Herbal Tea. The maximum amount of carotenoids is contained in the tea compositions based on black tea, the minimum quantity of carotenoids is contained in Sudanese rose Flower Tea. The minimum content of anthocyanins is characteristic for Mint Herbal Tea, and the maximum for Sudanese rose Flower Tea. Green Tea with Lemon and Ceylon Baikhovi Black Spiced Tea have similar levels of antioxidant bioavailability. After drinking both beverages, a significant increase in this indicator is noted in individuals with an initially low content of antioxidants in saliva. 
Antioxidants of Amaranth Phytotea are characterized by low level of bioavailability. Drinking of this beverage does not change the level of antioxidants in saliva regardless of their initial content. A cluster analysis of tea species on the content of special antioxidants was carried out.

\section{References}

1. J.-C. Preiser, JPEN J., 36(2), 147 (2012)

2. M. Breitenbach, P. Eckl, Biomolecules, 5, 1169 (2015)

3. A. A. Adwas et al., J. Appl. Biotechnol. Bioeng., 6(1), 43 (2019)

4. H. Nasri, A. Baradaran, H. Shirzad, M. Rafieian-Kopaei, Int. J. Prev. Med., 5, 1487 (2014)

5. P. Maslennikov, E. Golovina, A. Artemenko, Plants, 9(6), 746 (2020)

6. K. Jakubczyk et al., Foods, 9(4), 483 (2020)

7. P. Feduraev, L. Skrypnik, A. Riabova, P. Maslennikov, G. Chupakhina, Plants, 9(4), 476 (2020)

8. P. V. Maslennikov, G. N. Chupakhina, L. N. Skrypnik, P. V. Feduraev, A. S. Melnik, Russ. J. Ecol., 49(5), 384 (2018)

9. P. V. Maslennikov, G. N. Chupakhina, L. N. Skrypnik, P. V. Feduraev, A. S. Melnik, Int. J. of Environmental Studies, 75(5), 719 (2018)

10. J. Yang, R.H. Liu, IFST J., 48(1), 163 (2012)

11. S. Abdeltaif, K. A. SirElkhatim, Recycling, 3(2), 27 (2018)

12. S. Habtemariam, G. Varghese. Beverages, 1(3), 169 (2015)

13. A. Korus, Int. J. of Food Properties, 14(5), 1069 (2011)

14. A. Jaroszewska, W. Biel, Chilean J. of Agricultural Research, 77(2), 155 (2017)

15. G. N. Chupakhina, M. Shansky, A. Parol, N. Y. Chupakhina, P. V. Feduraev, L. N. Skrypnik, P. V. Maslennikov, Agronomy Research, 16, 1976 (2018) 